Article

\title{
Investigation of 1064-nm Pumped Type II SPDC in Potassium Niobate for Generation of High Spectral Purity Photon Pairs
}

\author{
Donghwa Lee, Ilhwan Kim and Kwang Jo Lee *(D)
}

check for

updates

Citation: Lee, D.; Kim, I.; Lee, K.J. Investigation of 1064-nm Pumped Type II SPDC in Potassium Niobate for Generation of High Spectral Purity Photon Pairs. Crystals 2021, 11, 599. https://doi.org/10.3390/ cryst11060599

Academic Editors: Yan Sheng and Katia Gallo

Received: 23 April 2021

Accepted: 24 May 2021

Published: 26 May 2021

Publisher's Note: MDPI stays neutral with regard to jurisdictional claims in published maps and institutional affiliations.

Copyright: (c) 2021 by the authors. Licensee MDPI, Basel, Switzerland This article is an open access article distributed under the terms and conditions of the Creative Commons Attribution (CC BY) license (https:/ / creativecommons.org/licenses/by/ $4.0 /)$.
Department of Applied Physics, Institute of Natural Science, Kyung Hee University, Yongin-si 17104, Korea; fairytale095@gmail.com (D.L.); dlfghks383@gmail.com (I.K.)

* Correspondence: kjlee88@khu.ac.kr

\begin{abstract}
The generation and detection of nonclassical light of about $2 \mu \mathrm{m}$ has good potential in an emerging field of high-sensitivity metrology, especially gravitational wave detection, as well as freespace quantum communication. A pair of photons is generated through a spontaneous parametric down-conversion (SPDC) process in a nonlinear optic crystal, which can be properly entangled in a spatial region where two beams with each polarization overlap or in a Sagnac-loop interferometer configuration. We investigated theoretically and numerically Type II SPDC in a potassium niobate $\left(\mathrm{KNbO}_{3}, \mathrm{KN}\right)$ crystal, which is useful as a material platform for generating photon pairs of high spectral purity in the 2- $\mu \mathrm{m}$ range. The technique is based on the frequency degenerate SPDC under Type II extended phase matching (EPM). We described the EPM characteristics of KN and showed that it is practically feasible for a 1064-nm pumped SPDC under moderate temperature conditions. The effective nonlinear optic coefficient of $\mathrm{KN}$ is at least four-times larger than those of other crystals using the Type II EPM approach, which implies a significant improvement in SPDC efficiency. The joint spectral analysis showed that a pair of photons can be generated with a high purity of 0.995 through proper pump filtering.
\end{abstract}

Keywords: potassium niobate; orthorhombic $\mathrm{mm}^{2}$ crystal; parametric down-conversion; photon-pair generation; extended phase matching

\section{Introduction}

The generation and detection of nonclassical light of about $2 \mu \mathrm{m}$ has good potential in an emerging field of high-sensitivity metrology, especially in gravitational wave detection [1-3]. Such long wavelengths can reduce quantum noise and scattering losses in interferometric gravitational wave detectors such as the laser interferometer gravitational wave observatory (LIGO)-Voyager [4]. Since this $2-\mu \mathrm{m}$ band belongs to a window of high transparency in the atmospheric absorption spectrum, quantum light sources in this band also have great potential for applications such as free space quantum communication and sensing, with the advantages of high sensitivity and low noise measurement [4-6]. Mansell et al. utilized a combination of a second-harmonic generator and an optical parametric oscillator with two pieces of periodically poled potassium titanyl phosphates (PPKTPs) to generate nonclassical squeezed states of light [1]. Prabhakar et al. demonstrated twophoton quantum interference and entanglement at $2.1 \mu \mathrm{m}$ using Type 0 spontaneous parametric down-conversion (SPDC) in a magnesium oxide-doped periodically poled lithium niobate (MgO:PPLN) [3]. In future studies, the basic requirements of the platform for generating photon pairs in the $2-\mu \mathrm{m}$ spectral range can be listed as follows: (1) SPDC using the frequency-degenerate Type II extended phase matching (EPM) is desirable for the generation of high spectral purity photon pairs. Here, the EPM refers to the simultaneous achievement of phase matching (PM) and group velocity $(\mathrm{GV})$ matching between interacting photons $[7,8]$. In addition, two photons generated simultaneously via Type II SPDC have polarization states that are perpendicular to each other. In this case, the 
polarization-entanglement can be obtained in the spatial region where two beams (or cones) with each polarization overlap, or in a Sagnac-loop interferometer configuration [9-11]. (2) A prevalent commercial laser is preferred as a light source for SPDC pumping to generate photon pairs in the $2-\mu \mathrm{m}$ band. Coherent light sources with a wavelength of about $1 \mu \mathrm{m}$ that are suitable for SPDC pumping include distributed feedback lasers, Fabry-Pérot lasers, and $\mathrm{Yb}$-doped fiber lasers, but the most widely used high power and stable lasers are solid-state $\mathrm{Nd}$ :YAG (neodymium-doped yttrium aluminum garnet, $\mathrm{Nd}: \mathrm{Y}_{3} \mathrm{Al}_{5} \mathrm{O}_{12}$ ) lasers. For example, 1064-nm Nd:YAG lasers are used in a variety of applications in science and technology, especially in materials processing, medicine, spectroscopy, and other laser pumping [12]. The wavelength of the photon pairs generated via the SPDC pumped by the Nd:YAG laser is $2128 \mathrm{~nm}$, which is within the high transparency range in the atmospheric absorption spectrum [5]. (3) The larger the nonlinearity of the crystal used for SPDC, the higher the SPDC efficiency. The SPDC efficiency is proportional to the square of the effective nonlinear optic coefficient for the given EPM condition [13]. However, the 1064-nm pumped SPDC of the Type II EPM approach has not yet been reported to the best of our knowledge. Now, it is timely to find suitable candidates for nonlinear optic crystals that satisfy all of the requirements listed above and investigate their usefulness as a generator of photon pairs in the $2-\mu \mathrm{m}$ band. In this paper, we theoretically and numerically investigate the Type II SPDC in a potassium niobate $\left(\mathrm{KNbO}_{3}, \mathrm{KN}\right)$ crystal, which is useful as a material platform for generating photon pairs of high spectral purity in the $2-\mu \mathrm{m}$ spectral range. The technique is based on the frequency degenerate SPDC under Type II EPM for the high spectral purity of the biphoton states. First, we investigate the Type II EPM characteristics of KN in terms of the beam propagation direction, the spectral position of photon pairs, and the corresponding effective nonlinearities and beam walk-offs. We will then show that Type II EPM is feasible for a 1064-nm pumped SPDC under a moderate temperature condition, which is followed by the comparison of the effective nonlinear optic coefficients $\left(d_{\text {eff }}\right)$ calculated under the given condition with those of other crystals using the Type II EPM. The result showed that the $d_{\text {eff }}$ values of $\mathrm{KN}$ is $4.67,4.03$, and 7.44-times larger than those of PPKTP, PPLN, and $\beta-\mathrm{BaB}_{2} \mathrm{O}_{4}(\mathrm{BBO})$, respectively. The joint spectral analysis showed that a pair of photons with a wavelength of $2128 \mathrm{~nm}$ can be generated by a 1064-nm pumped Type II SPDC, with a high purity of 0.995 through proper pump filtering.

\section{Materials and Theories}

$\mathrm{KN}$ is a negative biaxial crystal belonging to the point group of orthorhombic $\mathrm{mm}^{2}$ at room temperature, and its lattice constants are $a=0.56896 \mathrm{~nm}, b=0.39692 \mathrm{~nm}$, and $c=0.57256 \mathrm{~nm}[14,15]$. The crystallographic axes $(a, b, c)$ of $\mathrm{KN}$ are all perpendicular to each other and have a relationship of $(a, b, c)=(y, x, z)$ with the optical axes. In this case, the order of refractive-index (RI) magnitudes is given by $n_{z}<n_{y}<n_{x}$. The temperaturedependent Sellmeier equations of KN are found in [16]. Figure 1a illustrates Type II SPDC in a $\mathrm{KN}$, where an input pump photon with a frequency $2 \omega$ generates a pair of photons (signal and idler) with the same frequency $\omega$ and polarization states perpendicular to each other. The collinear birefringent PM (BPM) is achieved between the wave vectors (k) of the pump, signal, and idler photons as shown in Figure 1b. However, their beam directions (i.e., Poynting vectors, s) are generally not parallel to the crystallographic axes of $\mathrm{KN}$, resulting in a spatial walk-off due to the birefringence as shown in Figure 1c. This spatial walk-off will be discussed further in a later paragraph. The type II BPM condition can be expressed as:

$$
\Delta k=\left|k^{(l)}(2 \omega)-k^{(h)}(\omega)-k^{(l)}(\omega)\right|=0,
$$

where each $k$ represents the wave number of the interacting wave, and is defined as $k^{(m)}(j \omega)=(j \omega / c) n^{(m)}$. The unit $k$-vector is defined in a spherical coordinate as $(\sin \theta \cos \phi$, $\sin \theta \phi, \cos \theta)$. Here, $\theta$ and $\phi$ are the polar and azimuthal angles, respectively. The RIs of the 
two eigen-polarization modes of light traveling inside a $\mathrm{KN}$ are expressed as follows by solving the Fresnel equation of the wave normal [17]:

$$
n^{(m)}(j \omega)=\sqrt{\frac{2}{-B_{j} \pm \sqrt{B_{j}^{2}-4 C_{j}}}} .
$$

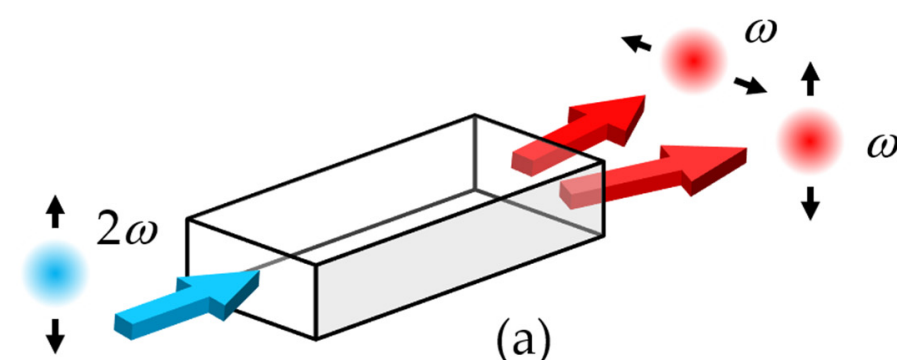

(a)

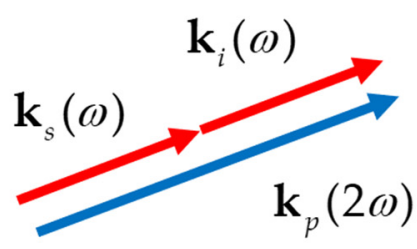

(b)

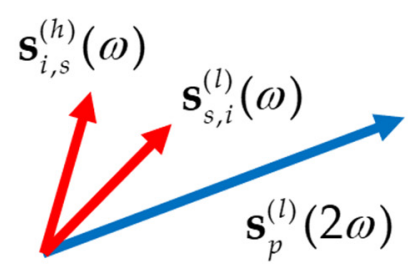

(c)

Figure 1. Schematic diagrams showing: (a) the relationships of frequencies and polarizations of pump, signal, and idler photons interacting via Type II spontaneous parametric down conversion (SPDC) in potassium niobate (KN), (b) the birefringent phase matching (BPM) between the wave vectors (k) of interacting waves, and (c) the spatial walk-off between the interacting beam lines described in the directions of Poynting vectors (s).

The parameters in Equation (2) are defined as follows:

$$
\begin{gathered}
B_{j}=-\left(b_{j}+c_{j}\right) k_{x}^{2}-\left(a_{j}+c_{j}\right) k_{y}^{2}-\left(b_{j}+a_{j}\right) k_{z}^{2}, \\
C_{j}=b_{j} c_{j} k_{x}^{2}+a_{j} c_{j} k_{y}^{2}+b_{j} a_{j} k_{z}^{2}, \\
a_{j}=n_{x}(j \omega), b_{j}=n_{y}(j \omega), c_{j}=n_{z}(j \omega),
\end{gathered}
$$

where, each $k_{i}$ represents the $x, y$, and $z$-axis components of the wave vector. $j$ can be 1 or 2 , then $n^{(m)}(2 \omega)$ and $n^{(m)}(\omega)$ denote the RIs of the pump wave and the signal (or idler) wave with frequencies $2 \omega$ and $\omega$, respectively. $m$ in Equation (2) can be either $l$ or $h$, representing low or high RI. $l$ and $h$ are obtained by taking plus and minus from the \pm sign of the denominator in Equation (2), respectively. The temporal walk-off between the interacting photons due to the difference in GV can be defined as the time delay $(\Delta T)$ per unit crystal length as follows:

$$
\frac{\Delta T}{L} \equiv \frac{\Delta n_{g}}{c}
$$

where $L$ and $\Delta n_{g}$ represent the crystal length and the group index difference between the interacting photons, respectively [18]. The GV matching for the Type II interaction is achieved when $\Delta n_{g}=0$ in Equation (6), which can be simplified as:

$$
2 n_{g}^{(l)}(2 \omega)=n_{g}^{(h)}(\omega)+n_{g}^{(l)}(\omega)
$$

Each subscript $g$ in Equation (7) represents the group index. Now, the Type II EPM shown in Figure $1 \mathrm{a}$ is defined as Equations (1) and (7) being satisfied simultaneously. Each of these equations is a function of three variables: the pump wavelength $\left(\lambda_{p}\right), \theta$, and $\phi$. Therefore, by solving a system of Equations (1) and (7) while changing $\lambda_{p}$, we can obtain the solution set of $\theta$ and $\phi$, i.e., the direction of pump wave vector for the Type II EPM at the given $\lambda_{p}$. Thus, by sweeping the pump wave vector along the direction characterized by the solution set of $\theta$ and $\phi$, we can continuously tune the resonant $\lambda_{p}$. This means that the spectral position of a photon pair can be selectively determined or tuned within the range of the solution sets while maintaining the high spectral purity of biphoton states. In contrast, for PPKN, Type II EPM can only be achieved at a single wavelength [18]. 
The effective nonlinear optic coefficients $\left(d_{e f f}\right)$ for a given direction of pump wave can generally be expressed as a linear combination of $d$-values as follows:

$$
d_{e f f}=\xi_{1} d_{15}+\xi_{2} d_{24}+\xi_{3} d_{31}+\xi_{4} d_{32}+\xi_{5} d_{33}
$$

where the five $d$-values of $\mathrm{KN}$ are $d_{15}=12.4 \pm 0.2 \mathrm{pm} / \mathrm{V}, d_{31}=11.9 \pm 0.2 \mathrm{pm} / \mathrm{V}$, $d_{24}=12.8 \pm 0.2 \mathrm{pm} / \mathrm{V}, d_{32}=13.7 \pm 0.2 \mathrm{pm} / \mathrm{V}$, and $d_{33}=20.6 \pm 0.2 \mathrm{pm} / \mathrm{V}$ at $1064 \mathrm{~nm}[19,20]$ Here, the coefficients $\xi_{i}$ are determined by the relationship between the crystallographic axes and the optical axes in a biaxial crystal with the point symmetry $\mathrm{mm}^{2}$ [21]. For a KN with the relationships of $(y, x, z)=(a, b, c)$, the $\xi$-coefficients are given as follows:

$$
\begin{gathered}
\left.\xi_{1}=-A E(B C E-G H)(B C H+G E)\right)-A H(B C E-G H)^{2}, \\
\xi_{2}=-A E(B G H-C E)(B G E+C H)-A H(B G E+C H)^{2}, \\
\xi_{3}=-A E(B C E-G H)(B C H+G E), \\
\xi_{4}=-A E(B G H-C E)(B G E+C H), \\
\xi_{5}=-A^{3} E^{2} H,
\end{gathered}
$$

where the angle-dependent parameters of $A, B, C, G, E$, and $H$ correspond to $\sin \theta, \cos \theta$, $\sin \phi, \cos \phi, \sin \delta$, and $\cos \delta$, respectively. The angle $\delta$ introduced for convenience only is defined as:

$$
\tan \delta \equiv \frac{2 B G C}{A^{2} \cot ^{2} V_{z}-B^{2} G^{2}+C^{2}},
$$

where $V_{z}$ represents the angle between the $z$-axis and the optic axis of biaxial birefringence. For biaxial crystals with a relationship $n_{z}<n_{y}<n_{x}$, such as $\mathrm{KN}, V_{z}$ is given by:

$$
\cos V_{z}=\frac{n_{x}\left(n_{y}^{2}-n_{z}^{2}\right)^{1 / 2}}{n_{y}\left(n_{x}^{2}-n_{z}^{2}\right)^{1 / 2}}
$$

Due to the RI dependence of $V_{z}, d_{\text {eff }}$ is given as a function of three variables $\lambda_{p}, \theta$, and $\phi$. Thus, $d_{\text {eff }}$ can be obtained by substituting the solution sets of $\lambda_{p}, \theta$, and $\phi$ that satisfy the Type II EPM (i.e., Equations (1) and (7)). The SPDC efficiency is proportional to the square of $d_{\text {eff }}$ for a given direction of beam propagation [13].

When a light beam travels inside a biaxial crystal with the point symmetry $\mathrm{mm}^{2}$, the spatial walk-off between the wave vector and the Poynting vector occurs within the crystal, as depicted in Figure 1b,c [22]. The walk-off angle $(\rho)$ can be expressed as:

$$
\tan \rho_{j}^{(m)} \equiv\left\{n^{(m)}(j \omega)\right\}^{2}\left[\left(\frac{k_{x}}{\left\{n^{(m)}(j \omega)\right\}^{-2}-a_{j}}\right)^{2}+\left(\frac{k_{y}}{\left\{n^{(m)}(j \omega)\right\}^{-2}-b_{j}}\right)^{2}+\left(\frac{k_{z}}{\left\{n^{(m)}(j \omega)\right\}^{-2}-c_{j}}\right)^{2}\right]^{-1 / 2},
$$

where, all parameters of Equation (12) are defined in Equations (2)-(5). For the Type II EPM in biaxial crystals such as a KN, the relationships of Poynting vectors (s) of the interacting waves are illustrated in Figure 1c, while the BPM is collinear as shown in Figure 1b. The low-RI signal (or idler) beam $\left(\mathbf{s}_{s, i}^{(l)}\right)$ is always placed between the high-RI idler (or signal) beam $\left(\mathbf{s}_{i, s}^{(h)}\right)$ and the low-RI pump beam $\left(\mathbf{s}_{p}^{(l)}\right)$ as shown in Figure 1c. In this case, the spatial walk-off between the interacting beams can be defined as the largest angle $(w)$ formed by the Poynting vectors of the high-RI idler (or signal) beam and the low-RI pump beam:

$$
\cos w=\cos \rho_{1}^{(h)} \cos \rho_{2}^{(l)} .
$$

Here, $w$ is also given as a function of three variables $\lambda_{p}, \theta$, and $\phi$, which can be obtained by substituting the solution sets of $\lambda_{p}, \theta$, and $\phi$ satisfying the Type II EPM into Equation (13). 
The maximum deviation between the interacting beams after passing through the crystal of length $L$ is expressed as:

$$
\Delta=L \tan w .
$$

The joint spectral analysis is commonly used to quantify the heralded-state spectral purity of the SPDC output, which includes the construction of the signal-idler joint spectral amplitude (JSA) and the calculation of the purity of biphoton state via Schmidt decomposition [23-25]. Here, the spectral purity is a parameter that describes the degree of spectral uncorrelation between the signal and the idler photons. The signal-idler biphoton state $|\psi\rangle$ produced via SPDC can be expressed as:

$$
|\psi\rangle=\int_{0}^{\infty} \int_{0}^{\infty} d \omega_{s} d \omega_{i} f\left(\omega_{s}, \omega_{i}\right) \hat{a}_{s}^{\dagger}\left(\omega_{s}\right) \hat{a}_{i}^{\dagger}\left(\omega_{i}\right)|0\rangle|0\rangle,
$$

where $\hat{a}_{s}^{\dagger}$ and $\hat{a}_{i}^{\dagger}$ are the creation operators of the signal and idler photons, respectively, and $\omega_{s}$ and $\omega_{i}$ are the corresponding frequencies. The correlation function, $f\left(\omega_{s}, \omega_{i}\right)$, represents the biphoton JSA, and is defined as the product of pump envelop (PE) function and the PM function as follows:

$$
f\left(\omega_{s}, \omega_{i}\right) \equiv N \alpha\left(\omega_{s}, \omega_{i}\right) \varphi\left(\omega_{s}, \omega_{i}\right) .
$$

Here, $N$ denotes the normalization constant. If a pump with Gaussian spectral shape is assumed, the PE function can be written as:

$$
\alpha\left(\omega_{s}, \omega_{i}\right) \propto \exp \left[-\frac{\left(\omega_{s}+\omega_{i}-\omega_{p}\right)^{2}}{\sigma_{p}^{2}}\right],
$$

where $\omega_{p}$ and $\sigma_{p}$ represent the center frequency and bandwidth of the pump, respectively. The PM function is given in the form of a sinc function as shown below:

$$
\varphi\left(\omega_{s}, \omega_{i}\right) \propto \operatorname{sinc}\left(\frac{\Delta k L}{2}\right)
$$

where $\Delta k$ represents the phase-mismatch defined in Equation (1). For the given direction of the pump wave that satisfies the Type II EPM (i.e., the solution sets of $\theta$ and $\phi$ ), Equation (18) is given as a function of the signal and idler wavelengths (or $\lambda_{s}$ and $\lambda_{i}$ ), so the JSA can be plotted on a two-dimensional plane as a function of $\lambda_{s}$ and $\lambda_{i}$. Then, the purity can be calculated via the following Schmidt decomposition:

$$
f\left(\omega_{s}, \omega_{i}\right)=\sum_{j} \sqrt{c_{j}}\left|\zeta_{s, j}\right\rangle\left|\zeta_{i, j}\right\rangle,
$$

where Schmidt coefficients, $c_{j}$, are a set of non-negative real numbers satisfying the normalization condition, $\sum_{j} c_{j}=1 .\left|\zeta_{s, j}\right\rangle$ and $\left|\zeta_{i, j}\right\rangle$ represent the orthonormal basis states called as Schmidt modes. Then the spectral purity, $P$, is defined as the sum of squares of Schmidt coefficients as [23-25]:

$$
P=\sum_{j} c_{j}^{2}
$$

Therefore, once we plot the JSA as functions of $\lambda_{s}$ and $\lambda_{i}$ in a two-dimensional plane, $P$ can be calculated via the Schmidt decomposition in Equation (20).

\section{Simulations and Discussion}

Figure 2a shows the BPM and GV matching properties of SPDC in a $\mathrm{KN}$ at $22{ }^{\circ} \mathrm{C}$. The blue and the cyan surfaces represent the BPM and GV surfaces calculated using Equations (1) and (7), respectively. The vertical axis represents the wavelength of the pump photon $\left(\lambda_{p}\right)$, and the base of the coordinates is the plane formed by the angles $\theta$ and $\phi$ indicating the direction of the pump wave vector. The intersection line of the two surfaces 
in Figure 2a spans a specific range characterized by $\lambda_{p}, \theta$, and $\phi$, which corresponds to the Type II EPM. This is more clearly plotted in Figure $2 b$. Here, the blue line represents the intersection of the two surfaces, and the green and red lines represent the projections of the blue line on the $\phi-\lambda_{p}$ and $\theta-\lambda_{p}$ planes, respectively. The range of $\lambda_{p}$ resonance that satisfies the Type II EPM is $1044.95-1062.78 \mathrm{~nm}$ at $22{ }^{\circ} \mathrm{C}$, which corresponds to the $\phi$-range $90-0^{\circ}$ and the $\theta$-range $41.0-63.8^{\circ}$ as plotted in Figure $2 \mathrm{~b}$. It shows that the Type II EPM is achievable for all azimuthal angles, whereas for polar angles only in a specific angular region. The type II EPM properties of KN are summarized in Table 1.
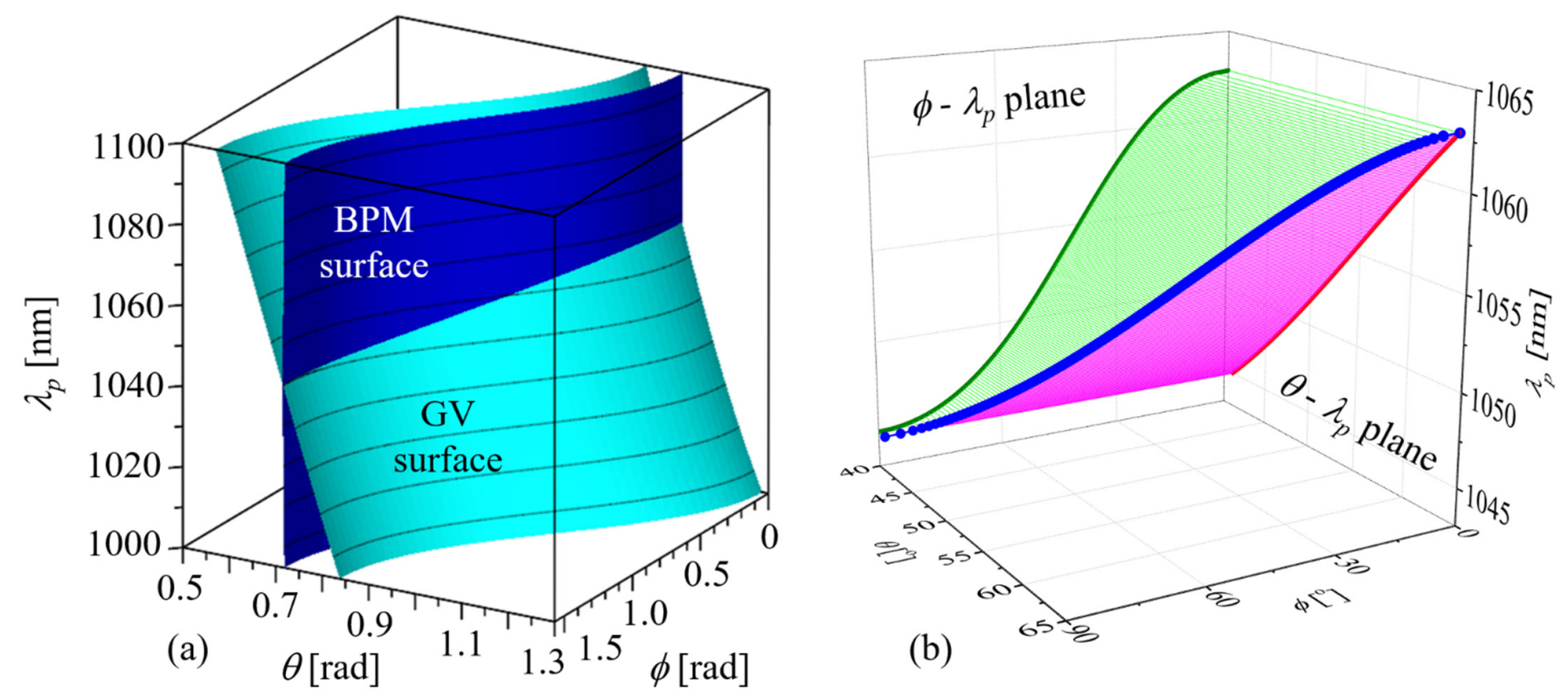

Figure 2. (a) Numerical simulation results of the BPM and the group velocity (GV) matching for SPDC in a $\mathrm{KN}$ at $22{ }^{\circ} \mathrm{C}$; (b) Type II extended phase matching (EPM) property of KN (the blue line) and its projections on the $\phi-\lambda_{p}$ and $\theta-\lambda_{p}$ planes (the green and red lines, respectively).

Table 1. The type II EPM properties of $\mathrm{KN}$ at $22{ }^{\circ} \mathrm{C}$ : the resonant pump wavelength $\left(\lambda_{p}\right)$ and the corresponding signal/idler wavelengths $\left(\lambda_{s}\right.$ and $\left.\lambda_{i}\right)$, the BPM direction $(\theta, \phi)$, the effective nonlinear optic coefficient $\left(d_{\text {eff }}\right)$, the spatial walk-off angle $(w)$ between the interacting beams, and the beam deviation $(\Delta)$.

\begin{tabular}{cc}
\hline Physical Quantities & Range of Values \\
\hline$\lambda_{p}(\mathrm{~nm})$ & $1044.95-1062.78$ \\
$\lambda_{s}\left(\right.$ or $\left.\lambda_{i}\right)(\mathrm{nm})$ & $2089.90-2125.55$ \\
$\phi\left(^{\circ}\right)$ & $90-0$ \\
$\theta\left(^{\circ}\right)$ & $41.00-63.80$ \\
$d_{e f f}(\mathrm{pm} / \mathrm{V})$ & $8.40-11.13$ \\
$w\left(^{\circ}\right)$ & $2.64-3.05$ \\
$\Delta(\mu \mathrm{m} / \mathrm{mm})$ & $46.10-53.29$ \\
\hline
\end{tabular}

The magnitude of the effective nonlinear optic coefficient $\left(d_{\text {eff }}\right)$ is determined by the BPM direction as described in the paragraphs with Equations (8)-(11). Since the efficiency of SPDC is proportional to the square of $d_{e f f}$, it is important to estimate the $d_{e f f}$-values for the given direction of wave vectors satisfying the Type II EPM. The $d_{\text {eff }}$-values were calculated numerically using Equations (8)-(11), and the results are plotted in Figure 3a as a function of $\lambda_{p}$. The horizontal width of the graph falls within the full range of $\lambda_{p}$ that satisfies the Type II EPM. In this range, the $d_{\text {eff }}$ values span $8.40-11.13 \mathrm{pm} / \mathrm{V}$, which are much larger than other cases using the Type II EPM approach, e.g., $d_{\text {eff }}=1.50 \mathrm{pm} / \mathrm{V}$ for BBO under the BPM; $2.39 \mathrm{pm} / \mathrm{V}\left(=(2 / \pi) d_{24}\right)$ for PPKTP and $2.77 \mathrm{pm} / \mathrm{V}\left(=(2 / \pi) d_{15}\right)$ for PPLN, under the 
first-order QPM [10,26,27]. The largest values of $d_{\text {eff }}$ are $11.13 \mathrm{pm} / \mathrm{V}$ at $1062.78 \mathrm{~nm}$, and the corresponding BPM direction is $(\theta, \phi)=\left(63.8^{\circ}, 0^{\circ}\right)$. It is noteworthy that the $d_{\text {eff-value }}$ is the largest when the wave vectors for the BPM are parallel to the $x-z$ plane $(\phi=0)$. The maximum walk-off angle $(w)$ between the interacting beams and the corresponding beam deviation $(\Delta)$ were calculated using Equations (13) and (14), respectively, and plotted together in Figure $3 \mathrm{~b}$ as a function of $\lambda_{p}$ that satisfies the Type II EPM. Over the whole range of $\lambda_{p}$, the calculated $w$ value spans $2.64-3.05^{\circ}$. The deviation $(\Delta)$ between the interacting beams per crystal of unit millimeter length is $46.10-53.29 \mu \mathrm{m} / \mathrm{mm}$, which can be sufficiently overcome by using a pump beam of large size in thick crystals. The calculated values of $d_{\text {eff }}, w$, and $\Delta$ are also summarized in Table 1.
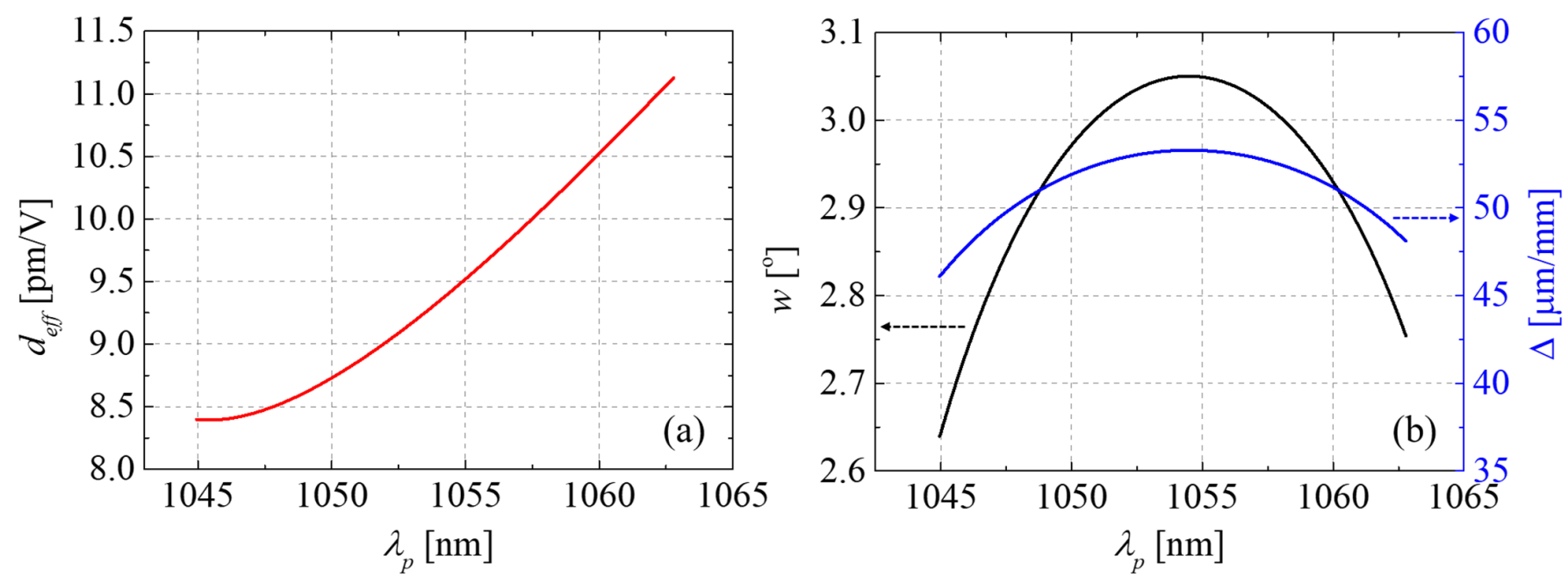

Figure 3. (a) The effective nonlinear optic coefficient $\left(d_{e f f}\right)$; (b) the spatial walk-off angle $(w)$ between the interacting beams, and the beam deviation $(\Delta)$. The numerical simulation results were plotted as functions of $\lambda_{p}$ satisfying the Type II EPM.

Now, for the case with the largest $d_{\text {eff }}$ value (i.e., $\phi=0$ ), the temperature behaviors of Type II EPM characteristics of KN are investigated. Figure 4a shows the change in $\lambda_{p}$ resonance and the corresponding polar angle $(\theta)$, as a function of temperature, $t$. As the temperature rises from 20 to $130{ }^{\circ} \mathrm{C}, \lambda_{p}$ shifts to longer wavelengths $(1062.68 \mathrm{~nm} \rightarrow$ $1070.06 \mathrm{~nm}$ ), while the polar angle for Type II EPM only increases by $2.6^{\circ}$ from $68.8^{\circ}$. Since the azimuthal angle is always $\phi=0$ for the case with the largest $d_{\text {eff }}$ value, only the polar angle needs to be fine-tuned to maintain Type II EPM while the temperature rises. At $t=45.59{ }^{\circ} \mathrm{C}$, the $\lambda_{p}$ resonance reaches $1064 \mathrm{~nm}$, which is the center wavelength of typical Nd:YAG lasers. This means that, without preparing a light source suitable for the resonance of the crystal for SPDC, high-purity photon pairs can be generated under moderate temperature conditions using a commercial high-power laser. High power SPDC pumping within the laser damage threshold of the crystal, along with the largest nonlinearity, can increase the maximum number of photon pairs per second. While the temperature rises from 20 to $130{ }^{\circ} \mathrm{C}$, the $d_{\text {eff }}$ value increases slightly from 11.12 to $11.36 \mathrm{pm} / \mathrm{V}$, as shown in Figure $4 \mathrm{~b}$. The $d_{\text {eff }}$ value of $\mathrm{KN}$ calculated at $t=45.59{ }^{\circ} \mathrm{C}$ (or $\lambda_{p}=1064 \mathrm{~nm}$ ) is $11.16 \mathrm{pm} / \mathrm{V}$, which is $4.67,4.03$, and 7.44-times larger than those of PPKTP, PPLN, and BBO using the Type II EPM approach, respectively. These values correspond to 21.80, 16.24, and 55.40-times increases in SPDC efficiency, respectively, because the nonlinear optic efficiency is proportional to the square of $d_{\text {eff }}$ [13]. Here, we note that the generation of photon pairs based on the Type II SPDC in the 2- $\mu \mathrm{m}$ band is also feasible in KTP isomorphs, but, in this case, 1064-nm pumping is not possible and the effective nonlinearity is much smaller than that of KN [28]. To the best of our knowledge, the generation of photon pairs based on the Type II EPM SPDC in biaxial birefringent crystals with orthorhombic $\mathrm{mm}^{2}$ point symmetry (e.g., $\mathrm{KN}$ and non-poled KTP isomorphs) has not yet been experimentally demonstrated, nor has the 1064-nm 
pumped case. We highlight that the generation of photon pairs is possible with high efficiency and high spectral purity based on 1064-nm pumped Type II SPDC in KN. We believe that $\mathrm{KN}$ can be the best candidate for this purpose. Experimental demonstrations are currently underway using commercially available bulk KN crystals [29]. The crystal most similar to $\mathrm{KN}$ in its constituent atoms is lithium niobate $\left(\mathrm{LiNbO}_{3}\right.$ or $\left.\mathrm{LN}\right)$. $\mathrm{LN}$ is "nonlinear optically" different from $\mathrm{KN}$ in that it belongs to the trigonal $3 \mathrm{~m}$ point group and exhibits uniaxial birefringence. Several studies on the photon-pair generation using PPLNs have been reported so far, but in all of the cases the EPM approach was not used or the frequencies of the generated photon pairs were not degenerated [30-34]. Experimental results for the generation of photon pairs in thin-film PPLN have only been reported recently, but again, Type 0 SPDC were used $[35,36]$. The frequency-degenerate, Type II EPM approach for LN has recently been reported only as a theoretical study [26]. However, for non-poled LNs, the effective nonlinearity (and thus the SPDC efficiency) is too low as $d_{e f f} \leq 0.2 \mathrm{pm} / \mathrm{V}$, and for PPLNs, the wavelength of the photon pair is longer than $3.5 \mu \mathrm{m}$ (with $d_{\text {eff }} \sim 4 \mathrm{pm} / \mathrm{V}$ ), which is far from the 2- $\mu \mathrm{m}$ band (or 1064-nm pumping). For a 1064-nm-pumped SPDC in KN, the spectral position of a photon pair is $2128 \mathrm{~nm}$, which is within the high transparency range in the atmospheric absorption spectrum [5]. The spatial walk-off angle $(w)$ between the interacting photons and the corresponding beam deviation $(\Delta)$ calculated using Equations (13) and (14), respectively, are $3.20^{\circ}$ and $55.89 \mu \mathrm{m} / \mathrm{mm}$, respectively. Since these values are smaller than those of BBO (i.e., $w=3.5^{\circ}$ and $\Delta=61.16 \mu \mathrm{m} / \mathrm{mm}$ ), which are widely used for the photon-pair generation, longer nonlinear interaction lengths within the crystals can be used to increase SPDC efficiency. For the case with the maximum $d_{e f f}$ value, the Type II EPM conditions at $\lambda_{p}=1064 \mathrm{~nm}$ are listed in Table 2.
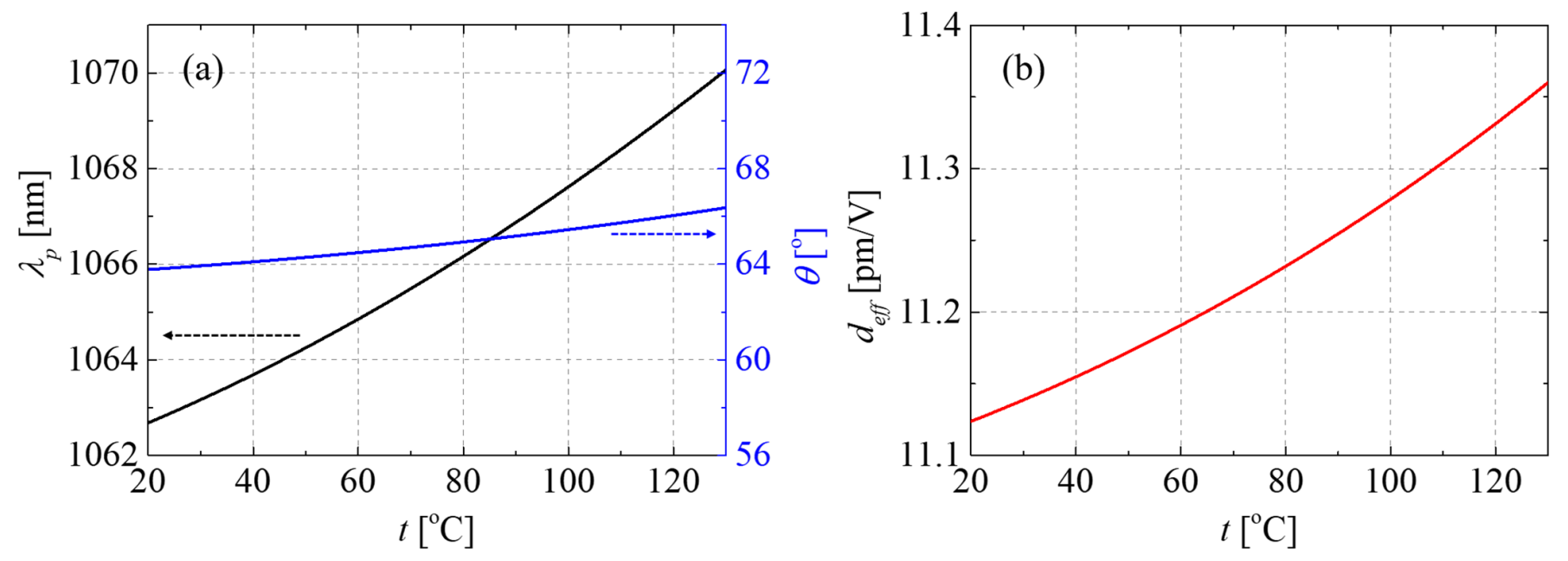

Figure 4. For the case with the largest $d_{e f f}$ value (i.e., $\phi=0$ ) under Type II EPM, the temperature dependence of: (a) the $\lambda_{p}$ resonance and the corresponding polar angle $(\theta)$ and $(\mathbf{b})$ the $d_{\text {eff }}$ values plotted as a function of $t$.

To quantify the heralded-state spectral purity of the SPDC output, we now analyze the signal-idler JSA properties, including the calculation of the purity of the biphoton state. Figure 5 shows the JSA characteristics of Type II SPDC for the case of KN listed in Table 2 (i.e., $\lambda_{p}=1064 \mathrm{~nm}$, the maximum $d_{e f f}$, Type II EPM). Figure $5 \mathrm{a}, \mathrm{b}$ shows density plots of the PE and PM functions calculated using Equations (17) and (18), respectively, for a pump with a linewidth of $2.43 \mathrm{~nm}$. This level of linewidth can be easily achieved through a combination of several filters placed in series because bandpass filters for pump filtering in the $1-\mu \mathrm{m}$ band are commercially available with a high technical level. The joint spectral intensity (JSI) calculated using Equation (16) is shown in Figure 5c, where JSI is defined as $|\mathrm{JSA}|^{2}$. Here, the crystal length of $10 \mathrm{~mm}$ is used for all calculations. All of the density plots in Figure $5 \mathrm{a}-\mathrm{c}$ are displayed in a $40 \mathrm{~nm} \times 40 \mathrm{~nm}$ window. The solid (blue) and dashed (red) lines in Figure $5 \mathrm{~d}$ represent the contour lines for JSI $=0.5$ and a circle with a diameter 
of $8.1 \mathrm{~nm}$, respectively. The contour line represents a circular shape, and the spectral purity calculated using Schmidt decomposition in Equation (20) reaches 0.995. The results show that at a wavelength of $2128 \mathrm{~nm}$, very high spectral purity photon pairs can be generated in KN via a 1064-nm pumped SPDC with a Type II EPM approach.

Table 2. For the case with the maximum $d_{\text {eff }}$ value, the Type II EPM conditions where the resonance of the pump photon is located at the center wavelength of Nd:YAG lasers (i.e., $1064 \mathrm{~nm}$ ).

\begin{tabular}{cc}
\hline Physical Quantities & Values \\
\hline$t\left({ }^{\circ} \mathrm{C}\right)$ & 45.59 \\
$\lambda_{p}(\mathrm{~nm})$ & 1064.00 \\
$\lambda_{s}\left(\right.$ or $\left.\lambda_{i}\right)(\mathrm{nm})$ & 2128.00 \\
$\phi\left(^{\circ}\right)$ & 0 \\
$\theta\left(^{\circ}\right)$ & 45.59 \\
$d_{e f f}(\mathrm{pm} / \mathrm{V})^{1}$ & 11.16 \\
$w\left({ }^{\circ}\right)$ & 3.20 \\
$\Delta(\mu \mathrm{m} / \mathrm{mm})$ & 55.89 \\
\hline
\end{tabular}

${ }_{1}$ cf. $d_{e f f}=1.50 \mathrm{pm} / \mathrm{V}$ for BBO under the BPM; $2.39 \mathrm{pm} / \mathrm{V}\left(=(2 / \pi) d_{24}\right)$ for PPKTP and $2.77 \mathrm{pm} / \mathrm{V}\left(=(2 / \pi) d_{15}\right)$ for PPLN, under the first-order QPM.
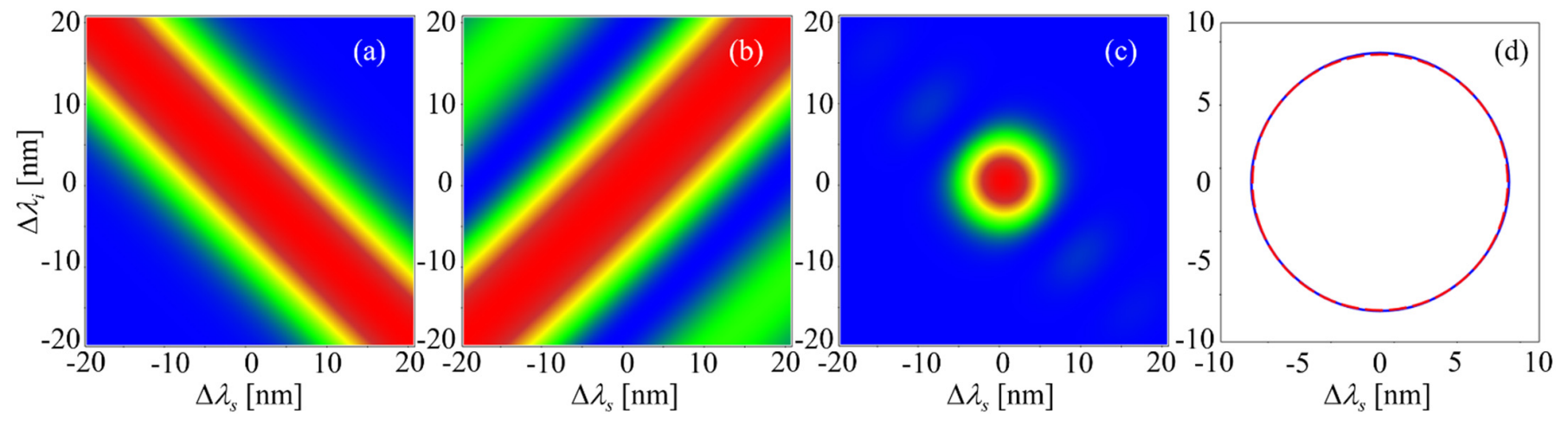

Figure 5. JSA characteristics of the SPDC output for the case of KN listed in Table 2 (i.e., $\lambda_{p}=1064$ nm, the maximum $d_{\text {eff }}$, Type II EPM): (a) the pump envelope (PE) function, (b) the phase matching (PM) function, (c) the joint spectral intensity (JSI), and (d) the contour line for JSI = 0.5 (blue solid line) shown with a circle with a diameter of $8.1 \mathrm{~nm}$ (red dashed line). The spectral purity calculated using Schmidt decomposition is 0.995 .

\section{Conclusions}

We have theoretically and numerically investigated the Type II SPDC properties of $\mathrm{KN}$. We theoretically described the Type II EPM characteristics of KN, in terms of BPM, GV matchings, effective nonlinearities, and spatial walk-offs between the interacting photons. The numerical simulation results showed that the spectral position of photon pairs can be tuned within the range of $2089.90-2125.55 \mathrm{~nm}$ by sweeping the pump wave vector along the direction satisfying the Type II EPM condition at $22{ }^{\circ} \mathrm{C}$. As the temperature of the $\mathrm{KN}$ rises from 20 to $130{ }^{\circ} \mathrm{C}$, the pump wavelength that satisfies the Type II EPM shifts from 1062.68 to $1070.06 \mathrm{~nm}$. The maximum effective nonlinearity (or SPDC efficiency) could be achieved when the wave vectors for the BPM are parallel to the $x-z$ plane. For the case with the largest $d_{\text {eff }}$, the Type II EPM is practically feasible for a $1064-\mathrm{nm}$ pumped SPDC at $t=45.59^{\circ} \mathrm{C}$. In this case, the spectral position of a photon pair is $2128 \mathrm{~nm}$, which is within the high transparency range in the atmospheric absorption spectrum. The photon-pair source in this band has great potential for applications such as free space quantum communication and sensing, with the advantages of high sensitivity and low noise measurement. The $d_{\text {eff }}$ value of $\mathrm{KN}$ is 4.67, 4.03, and 7.44-times larger than those of PPKTP, PPLN, and BBO, respectively, which means a significant improvement in SPDC efficiency. The heralded-state spectral purity of SPDC output was quantified via JSA analysis, and the results show that the purity calculated for a pump with a 2.43-nm bandwidth is as high as 0.995 . 
Author Contributions: Conceptualization, K.J.L.; Simulation, D.L.; writing-original draft preparation, D.L. and I.K.; writing-review and editing, K.J.L.; supervision, K.J.L. All authors have read and agreed to the published version of the manuscript.

Funding: This research was funded by National Research Foundation of Korea (Grant NRF-2019R1F1A1063937; Korea Institute of Science and Technology (Grant 2E29580-19-147); Institute for Information \& communications Technology Promotion (IITP) grant funded by the Korea government (MSIT) (No. 2020-0-00947).

Institutional Review Board Statement: Not applicable.

Informed Consent Statement: Not applicable.

Data Availability Statement: The data presented in this study are available in this article.

Conflicts of Interest: The authors declare no conflict of interest.

\section{References}

1. Mansell, G.L.; McRae, T.G.; Altin, P.A.; Yap, M.J.; Ward, R.L.; Slagmolen, B.J.J.; Shaddock, D.A.; McClelland, D.E. Observation of squeezed light in the $2 \mu \mathrm{m}$ region. Phys. Rev. Lett. 2018, 120, 203603. [CrossRef]

2. Shields, T.; Prabhakar, S.; Powell, D.; Taylor, G.G.; Morozov, D.; Ebrahim, M.; Kues, M.; Caspani, L.; Gawith, C.; Hadfield, R.H.; et al. Photon pair generation at $2080 \mu \mathrm{m}$ by down-conversion. In Proceedings of the 2019 Conference on Lasers and Electro-Optics Europe \& European Quantum Electronics Conference (CLEO/Europe-EQEC), Munich, Germany, 23-27 June 2019.

3. Prabhakar, S.; Shields, T.; Dada, A.C.; Ebrahim, M.; Taylor, G.G.; Morozov, D.; Erotokritou, K.; Miki, S.; Yabuno, M.; Terai, H.; et al. Two-photon quantum interference and entanglement at $2.1 \mu \mathrm{m}$. Sci. Adv. 2020, 6, eaay5195. [CrossRef]

4. Steinlechner, J.; Martin, I.W.; Bell, A.S.; Hough, J.; Fletcher, M.; Murray, P.G.; Robie, R.; Rowan, S.; Schnabel, R. Silicon-based optical mirror coatings for ultrahigh precision metrology and sensing. Phys. Rev. Lett. 2018, 120, 263602. [CrossRef]

5. Gemini Observatory. Available online: https://www.gemini.edu/observing/telescopes-and-sites/sites\#Transmission (accessed on 16 April 2021).

6. Kaushal, H.; Kaddoum, G. Optical communication in space: Challenges and mitigation techniques. IEEE Commun. Surv. Tutor. 2017, 19, 57-96. [CrossRef]

7. Giovannetti, V.; Maccone, L.; Shapiro, J.H.; Wong, F.N.C. Extended phase-matching conditions for improved entanglement generation. Phys. Rev. A 2002, 66, 043813. [CrossRef]

8. Kuzucu, O.; Fiorentino, M.; Albota, M.A.; Wong, F.N.C.; Kärtner, F.X. Two-photon coincident-frequency entanglement via extended phase matching. Phys. Rev. Lett. 2005, 94, 083601. [CrossRef] [PubMed]

9. Kwiat, P.; Mattle, K.; Weinfurter, H.; Zeilinger, A.; Sergienko, A.V.; Shih, Y. New high-intensity source of polarization-entangled photon pairs. Phys. Rev. Lett. 1995, 75, 4337-4341. [CrossRef]

10. Jin, R.-B.; Shimizu, R.; Wakui, K.; Fujiwara, M.; Yamashita, T.; Miki, S.; Terai, H.; Wang, Z.; Sasaki, M. Pulsed Sagnac polarizationentangled photon source with a PPKTP crystal at telecom wavelength. Opt. Express 2014, 22, 11498-11507. [CrossRef] [PubMed]

11. Weston, M.M.; Chrzanowski, H.M.; Wollmann, S.; Boston, A.; Ho, J.; Shalm, L.K.; Verma, V.B.; Allman, M.S.; Nam, S.W.; Patel, R.B.; et al. Efficient and pure femtosecond-pulse-length source of polarization-entangled photons. Opt. Express 2016, 24, 10869-10879. [CrossRef] [PubMed]

12. Koechner, W. Solid-State Laser Engineering, 6th ed.; Springer: New York, NY, USA, 2006; pp. 54-61.

13. Boyd, R.W. Nonlinear Optics, 4th ed.; Academic Press: San Diego, CA, USA, 2020; pp. 70-73.

14. Uematsu, Y. Nonlinear optical properties of $\mathrm{KNbO}_{3}$ single crystals in the orthorhombic phase. Jpn. J. Appl. Phys. 1974, 13, 1362-1368. [CrossRef]

15. Zysset, B.; Biaggio, I.; Günter, P. Refractive indices of orthorhombic $\mathrm{KNbO}_{3}$. I. Dispersion and temperature dependence. J. Opt. Soc. Am. B 1992, 9, 380-386. [CrossRef]

16. Jundt, D.H.; Gunter, P.; Zysset, B. A temperature-dependent dispersion equation for $\mathrm{KNbO}_{3}$. Nonlinear Opt. 1993, 4, $341-345$.

17. Yariv, A.; Yeh, P. Photonics: Optical Electronics in Modern Communications, 6th ed.; Oxford University Press: New York, NY, USA, 2007; pp. 30-33.

18. Lee, K.J.; Lee, S.; Shin, H. Extended phase matching properties of periodically poled potassium niobate crystals for mid-infrared polarization-entangled photon-pair generation. Appl. Opt. 2016, 55, 9791-9796. [CrossRef]

19. Baumert, J.-C.; Hoffnagle, J.; Günter, P. Nonlinear optical effects in $\mathrm{KNbO}_{3}$ crystals at $\mathrm{Al}_{x} \mathrm{Ga}_{1-x}$ As, dye, ruby and Nd:YAG laser wavelengths. Proc. SPIE 1984, 492, 374-385.

20. Roberts, D.A. Simplified characterization of uniaxial and biaxial nonlinear optical crystals: A plea for standardization of nomenclature and conventions. IEEE J. Quantum Electron. 1992, 28, 2057-2074. [CrossRef]

21. Dmitriev, V.G.; Nikogosyan, D.N. Effective nonlinearity coefficients for three-wave interactions in biaxial crystals of $\mathrm{mm}^{2}$ point group symmetry. Opt. Commun. 1993, 95, 173-182. [CrossRef]

22. Brehat, F.; Wyncke, B. Calculation of double-refraction walk-off angle along the phase-matching directions in nonlinear biaxial crystals. J. Phys. B At. Mol. Opt. Phys. 1989, 22, 1891-1898. [CrossRef] 
23. Grice, W.P.; Walmsley, I.A. Spectral information and distinguishability in type-II down-conversion with a broadband pump. Phys. Rev. A 1997, 56, 1627. [CrossRef]

24. Law, C.K.; Walmsley, I.A.; Eberly, J.H. Continuous frequency entanglement: Effective finite Hilbert space and entropy control. Phys. Rev. Lett. 2000, 84, 5304. [CrossRef] [PubMed]

25. Mosley, P.J.; Lundeen, J.S.; Smith, B.J.; Walmsley, I.A. Conditional preparation of single photons using parametric downconversion: A recipe for purity. New J. Phys. 2008, 10, 093011. [CrossRef]

26. Kim, I.; Lee, D.; Lee, K.J. Study of Type II SPDC in lithium niobate for high spectral purity photon pair generation. Crystals 2021, 11, 406. [CrossRef]

27. Shoji, I.; Nakamura, H.; Ohdaira, K.; Kondo, T.; Ito, R.; Okamoto, T.; Tatsuki, K.; Kubota, S. Absolute measurement of second-order nonlinear-optical coefficients of $\beta-\mathrm{BaB}_{2} \mathrm{O}_{4}$ for visible to ultraviolet second-harmonic wavelengths. J. Opt. Soc. Am. B 1999, 16, 620-624. [CrossRef]

28. Kim, I.; Lee, D.; Lee, K.J. Numerical investigation of high-purity polarization-entangled photon-pair generation in non-poled KTP isomorphs. Appl. Sci. 2021, 11, 565. [CrossRef]

29. CRYLINK. Available online: https://www.laser-crylink.com/laser-products/nonlinear-crystal/knbo3 (accessed on $20 \mathrm{May}$ 2021).

30. Fujii, G.; Namekata, N.; Motoya, M.; Kurimura, S.; Inoue, S. Bright narrowband source of photon pairs at optical telecommunication wavelengths using a type-II periodically poled lithium niobate waveguide. Opt. Express 2007, 15, 12769-12776. [CrossRef]

31. Martin, A.; Issautier, A.; Herrmann, H.; Sohler, W.; Ostrowsky, D.B.; Alibart, O.; Tanzilli, S. A polarization entangled photon-pair source based on a type-II PPLN waveguide emitting at a telecom wavelength. New J. Phys. 2010, 12, 103005. [CrossRef]

32. Cheng, X.; Sarihan, M.C.; Chang, K.-C.; Lee, Y.S.; Laudenbach, F.; Ye, H.; Yu, Z.; Wong, C.W. Design of spontaneous parametric down-conversion in integrated hybrid $\mathrm{Si}_{\mathrm{x}} \mathrm{N}_{\mathrm{y}}$-PPLN waveguides. Opt. Express 2019, 27, 30773-30787. [CrossRef]

33. Kuo, P.S.; Verma, V.B.; Nam, S.W. Demonstration of a polarization-entangled photon-pair source based on phase-modulated PPLN. OSA Contin. 2020, 3, 295-304. [CrossRef]

34. Kuo, P.S.; Gerrits, T.; Verma, V.; Nam, S.W.; Slattery, O.; Ma, L.; Tang, X. Characterization of type-II spontaneous parametric down-conversion in domain-engineered PPLN. Proc. SPIE Adv. Photonics Quantum Comput. Mem. Commun. 2016, 9762, 976211.

35. Zhao, J.; Ma, C.; Rüsing, M.; Mookherjea, S. High quality entangled photon pair generation in periodically poled thin-film lithium niobate waveguides. Phys. Rev. Lett. 2020, 124, 163603. [CrossRef]

36. Chen, J.-Y.; Sua, Y.M.; Ma, Z.-H.; Tang, C.; Li, Z.; Huang, Y.-P. Efficient parametric frequency conversion in lithium niobate nanophotonic chips. OSA Contin. 2019, 2, 2914-2924. [CrossRef] 